\title{
EVALUACIÓN DEL ESTADO NUTRICIONAL DE NIÑOS EN EDAD ESCOLAR DE DOS LOCALIDADES INDÍGENAS DE OAXACA.
}

\author{
NUTRITIONAL CONDITION EVALUATION OF CHILDREN IN SCHOOL AGE OF TWO INDIGENOUS \\ LOCATIONS OF OAXACA. \\ Luna-Hernández Jorge Fernando*, Ramírez-Díaz María del Pilar*, Guerrero-Contreras Israel*, \\ Guevara-Santillán Rosario*, Marín-Velázquez Juanelly*, Jiménez-Avendaño Erika*. \\ *Universidad del Istmo (UNISTMO), Campus Juchitán de Zaragoza, Oaxaca. México.
}

Citation: Luna-Hernández J.F., Ramírez-Díaz M.P., Guerrero-Contreras I., Guevara-Santillán R., Marín-Velázquez J., Jiménez-Avendaño E. (2020) Evaluación del estado nutricional de niños en edad escolar de dos localidades indígenas de Oaxaca. Revista Salud Pública y Nutrición, 19 (1), 1-9.

Editor: Esteban G. Ramos Peña, Dr. CS., Universidad Autónoma de Nuevo León, Facultad de Salud Pública y Nutrición, Monterrey Nuevo León, México. Copyright: (02020 Luna-Hernández J.F. et al. This is an open-access article distributed under the terms of Creative Commons Attribution License [CC BY 4.0], which permits unrestricted use, distribution, and reproduction in any medium, provided the original author and source are credited.

Competing interests: The authors have declared that no competing interests exist.

DOI: https://doi.org/10.29105/respyn19.1-1

Recibido: 28 de noviembre 2019; Aceptado: 17 de febrero 2020

Email: jorgeluna.900909@gmail.com 


\title{
EVALUACIÓN DEL ESTADO NUTRICIONAL DE NIÑOS EN EDAD ESCOLAR DE DOS LOCALIDADES INDÍGENAS DE OAXACA.
}

\author{
Luna-Hernández Jorge Fernando*, Ramírez-Díaz María del Pilar*, Guerrero-Contreras Israel*, Guevara- \\ Santillán Rosario*, Marín-Velázquez Juanelly*, Jiménez-Avendaño Erika*. \\ *Universidad del Istmo (UNISTMO), Campus Juchitán de Zaragoza, Oaxaca. México.
}

RESUMEN

Introducción. La malnutrición es un problema grave en la edad escolar y se polariza en excesos o carencias perjudicando física, psicológica y fisiológicamente la salud de los niños a corto y largo plazo. Objetivo: Conocer el estado nutricional de escolares de dos localidades indígenas de Oaxaca. Material y Método: Se realizó un estudio transversal observacional en 120 niños escolares de 6 a 12 años de las localidades de Progreso Guevea y Xadani Guevea de Humboldt, Oaxaca. El estado nutricio se determinó a través del puntaje z del IMC para la edad y talla para la edad en STATA V. 14. El análisis de los datos se realizó por medio de frecuencias, porcentajes, diferencias de medias y pruebas de Chi-cuadrada, $T$ de Student y $U$ de Mann-Whitney en SPSS V. 24. Resultados De los 120 niños analizados se obtuvo una prevalencia de sobrepeso y obesidad de $15 \%$ y de delgadez de $16.7 \%$. Se encontró asociación significativa en cuanto a la delgadez en la localidad de Xadani 4.10 (IC 95\% 1.13-14.91) y al estratificar por grado escolar en ambas comunidades se encontró mayor proporción de delgadez en escolares de $4^{\circ}$ a $6^{\circ}$ grado de Xadani $(p=0.001)$. Conclusiones: La doble carga de la malnutrición sigue permeando diferentes zonas indígenas; no obstante, su distribución no es uniforme en localidades con características semejantes, fenómeno que deberá de profundizarse para conocer sus causas.

Palabras Clave: Malnutrición, Estado nutricional, Escolares

\section{ABSTRACT}

Introduction: Malnutrition is a serious problem in school age and is polarized in excesses or deficiencies that can physically, psychologically and physiologically harm the health of children in the short and long term. Objective: To know the nutritional status of school children from two indigenous localities in Oaxaca Material and method: An observational cross-sectional study was conducted in 120 school children aged 6 to 12 years from the towns of Progreso Guevea and Xadani Guevea de Humboldt, Oaxaca. Weight and height were obtained. The nutritional status was determined through the $\mathrm{z}$ score of the BMI for age and height for age in STATA V. 14. The data was analyzed by means of frequencies, percentages, media differences and tests of X2, Student's T and Mann's U -Whitney in SPSS V. 24. Results: Of the 120 children analyzed, a prevalence of overweight and obesity of $15 \%$ and thinness of $16.7 \%$ was obtained. A significant association was found in terms of thinness in the locality of Xadani 4.10 (95\% Cl 1.13-14.91) and when stratifying by grade level in the communities, a higher proportion of thinness was found in students in grades 4-6 of Xadani $(p=0.001)$. Conclusions: The double burden of malnutrition continues to permeate different indigenous areas; However, its distribution is not uniform in localities with similar characteristics, a phenomenon that must be deepened to know its causes.

Key words: Malnutrition, Nutritional status, School children 


\section{Introducción}

La alimentación y la correcta nutrición en todas las etapas de la vida son fundamentales, principalmente en etapas tempranas del ser humano para el óptimo crecimiento y desarrollo fisiológico (Mameli et al., 2016). La malnutrición abarca un espectro que se polariza en deficiencia y exceso como desnutrición, sobrepeso (SP) y obesidad (OB) en donde la población infantil es uno de los grupos vulnerables para presentar cualquiera de sus expresiones (Kruger et al., 2012). Mientras que las deficiencias nutrimentales afectan el crecimiento y desarrollo en esta etapa, el SP y la OB en etapas pediátricas aumentan el riesgo de que estos problemas se mantengan hasta la edad adulta, impactando de manera significativa en el aspecto físico y fisiológico con problemas osteoarticulares; metabólicos como diabetes, síndrome metabólico y enfermedades cardiacas; psicológicos como bajo rendimiento escolar, depresión, ansiedad y un claro impacto en la calidad de vida. (Pollit, 1995; Bouma, 2017; Badillo, 2017; Dinkel, 2018).

La detección de malnutrición en población pediátrica se lleva a cabo a través de la comparación de mediciones antropométricas con los indicadores relacionados con la edad, talla, peso y sexo postulados por la Organización Mundial de la Salud (OMS) (Bouma, 2017). Existen distintos indicadores para analizar el estado nutricional de un niño, sin embargo, de acuerdo con el Fondo de las Naciones Unidas para la Infancia (UNICEF), el Índice de Masa Corporal (IMC) para la edad (IMC/Edad) es el indicador más adecuado para la detección de problemas relacionados a malnutrición en niños escolares. (UNICEF, 2012).

Se sabe que actualmente se han producido cambios significativos en el estilo y calidad de vida de los diferentes grupos poblacionales, principalmente en materia de alimentación; por un lado la industrialización ha propiciado que la disponibilidad de los alimentos densamente energéticos se globalice en mayor escala, con el consecuente impacto en los hábitos alimenticios y en el estado de salud como lo son el SP y la OB ; en contraparte sigue existiendo una alta prevalencia mundial de desnutrición y otro tipo de síndromes por la falta de accesibilidad y disponibilidad alimentaria y nutricional. Esta doble carga de morbilidad se observa particularmente en países en vías de desarrollo como México (Shamah, 2015; UNICEF, 2016;).

Este contexto involucra factores de riesgo para Enfermedades Crónicas No Transmisibles (ECNT) que se adoptan en la edad infantil y que pueden tener como consecuencia cualquier tipo de malnutrición, siendo un problema actual de salud pública de escala mundial y que aumenta el riesgo de mortalidad hasta en un 50\%. (Kruger et al., 2012; Macari, 2018; Endris, 2017). De acuerdo con los resultados de la Encuesta Nacional de Salud y Nutrición (ENSANUT) entre 2012 y 2016, se observó un incremento en la prevalencia de SP y OB en niñas escolares y una disminución en los niños del mismo grupo de edad. También se ha visto una tendencia por el aumento de peso en mujeres de zonas rurales, lo que puede indicar una posible transición nutricional y epidemiológica de las zonas más marginadas. (Shammah, 2018).

Es importante mencionar que la malnutrición es de etiología multidimensional, en la cual participan diversos factores sociales, políticos, económicos, culturales y ambientales, los cuales determinarán la presencia o ausencia de daños a la salud y su gravedad.

Desde esta perspectiva y de acuerdo con el Programa para el Desarrollo de Zonas Prioritarias (PDZP) de la Secretaría de Desarrollo Social (SEDESOL) el estado de Oaxaca es uno de los más pobres de México, cuenta con un número considerable de municipios con alto grado de marginación y de rezago social, así como la falta de infraestructura en salud suficiente y adecuada para dar cobertura a la población total, perjudicando en mayor medida a aquellas comunidades y municipios más alejados y más pobres. (SEDESOL,2013)

Por ello, es importante priorizar acciones enfocadas a la mejora de la salud en aquellos grupos de poblaciones que son más vulnerables, entre ellos la población indígena, ya que, al tener oportunidades económicas limitadas, la probabilidad de morbimortalidad aumenta, por ello, la prevención se vuelve esencial para mitigar las complicaciones del proceso salud-enfermedad. (Arcaya, Arcaya y Subramanian, 2015). Desde esta premisa el objetivo general del presente estudio fue conocer el estado nutricional de escolares de 6 a 12 años de dos localidades indígenas 
del municipio de Guevea de Humbolt, Oaxaca y de manera específica comparar el estado nutricional de ambas localidades, ya que se considera uno de los municipios indígenas con mayor índice de marginación.

\section{Material y Método \\ Se realizó un estudio observacional transversal utilizando el universo de niños escolares de dos localidades indígenas de Guevea de Humbolt (Xadani Guevea y Progreso Guevea de Humbolt). No se calculó muestra ya que son comunidades pequeñas, Xadani cuenta con 455 habitantes de los cuales el $61.9 \%$ corresponde a población indígena y Progreso registra 311 habitantes con un $78.4 \%$ de población indígena (SEDESOL, 2013; Instituto Nacional de Pueblos Indígenas[INPI],2015) por lo cual el universo de niños en edad escolar de 6 a 12 años se logró localizar en la única escuela primaria de cada una de las localidades (Escuela primaria Niños Héroes y Ricardo Flores Magón), reforzando con la información obtenida de los centros de salud. En total se identificó un universo de 120 niños en edad escolar.}

El muestreo se realizó por caso consecutivo mediante el listado de niños inscritos en las dos escuelas primarias. Los criterios de inclusión fueron escolares de 6 a 12 años que asistieran a la escuela primaria de cada una de las localidades y que se contara con la aprobación del estudio por medio del consentimiento y asentimiento informado. Los criterios de exclusión fueron aquellos niños con compromisos anatómicos, y aquellos cuyos padres no firmaran el consentimiento informado. Para ello, en primera instancia, se convocó a una junta con los padres de familia de ambas escuelas para explicar detalladamente el proceso y que pudieran firmar la carta de consentimiento informado con base en la declaración de Helsinki y con la autorización de las instituciones educativas de cada localidad. Asimismo, se les concedió el asentimiento informado a los niños con el objetivo de que pudieran decidir participar o no en el estudio. Los criterios de exclusión fueron aquellos niños cuyos padres no firmaran el consentimiento, que hayan faltado a la escuela el día de la evaluación o que tuvieran algún compromiso anatómico que dificultara su participación.
La evaluación antropométrica que comprendió la recolección de talla y peso fue realizada por una estudiante de la Licenciatura en Nutrición de la Universidad del Istmo, Juchitán. Para la toma de peso y estatura se les pidió a los niños cumplir las indicaciones, según lo establecido en el manual de Antropometría de Registro Nacional de Peso y Talla en Escolares 2015-2016 del Instituto Nacional de Ciencias Médicas y Nutrición Salvador Zubirán. (Ávila, et al., 2015).

Se utilizó una báscula Baurer G-20 con 8 indicadores para la toma del peso corporal en kilogramos y un estadímetro portátil seca 213 para la toma de la estatura en centímetros. Para el análisis del estado nutricional se consideró el indicador IMC para la edad, (IMC/E) determinando el puntaje $\mathrm{z}$ y su diagnóstico mediante el paquete estadístico STATA versión 14 (StataCorp, 2015) a través de las referencias de la OMS y de la misma manera se calculó la talla para la edad (Talla/E).

La información recolectada fue procesada en el paquete estadístico SPSS versión 24(IBM Corp, 2016). Para las variables cualitativas se obtuvieron frecuencias absolutas y porcentajes, en cuanto a las variables cuantitativas se calcularon medias y desviaciones estándar; para las pruebas de hipótesis, se utilizó Chi-cuadrada para variables cualitativas y $\mathrm{T}$ de student y U de Mann-Whitney para variables cuantitativas. Estos análisis se hicieron por sexo, localidad y grado escolar para el cual se crearon dos grupos de comparación; grupo 1 incluyendo a escolares de $1^{\circ}$ a $3^{\circ}$ grado y grupo 2 de $4^{\circ}$ a $6^{\circ}$ grado. Asimismo, se agruparon todos los grados de delgadez como una sola categoría para un mejor análisis del estado nutricional.

Para la estructura del documento y la comunicación de resultados se tomó en cuenta lo estipulado por la guía STROBE.

\section{Resultados}

Se evaluaron dos localidades con un total de 120 escolares, de los cuales el $62.5 \%$ (75) correspondía a la localidad de Xadani y el resto a la comunidad de Progreso 37.5\% (45). Dentro del total el $60 \%$ (72) lo conformaron niñas y $40 \%$ (48) fueron niños. De manera general se encontró una proporción conjunta de sobrepeso y obesidad (SP+OB) de $15 \%$ y de delgadez en todas sus formas de $16.7 \%$. 
Respecto a la comparación del estado nutricional por medio de antropometría entre comunidades específicamente por el indicador Talla/E el mayor porcentaje en ambas escuelas se encontró en normalidad, mientras que de acuerdo con el IMC/E se observó una diferencia estadísticamente significativa $(p<0.05)$; siendo la comunidad de Xadani quien presentó mayor proporción de delgadez (Tabla 1).

Tabla 1. Diagnóstico nutricional general y por localidad

\begin{tabular}{|c|c|c|c|c|c|c|}
\hline \multirow{2}{*}{ Estado nutricional - } & \multicolumn{2}{|c|}{ Total } & \multicolumn{2}{|c|}{ Xadani } & \multicolumn{2}{|c|}{ Progreso } \\
\hline & 120 & $\%$ & 75 & $\%$ & 45 & $\%$ \\
\hline \multicolumn{7}{|l|}{ Talla/Edad $^{\mathrm{a}}$} \\
\hline Talla baja & 4 & 3.3 & 3 & 4 & 1 & 2.2 \\
\hline Normal & 115 & 95.8 & 71 & 94.7 & 44 & 97.8 \\
\hline Muy alto & 1 & 1.3 & 1 & 1.3 & 0 & 0 \\
\hline \multicolumn{7}{|l|}{ IMC/Edad } \\
\hline Delgadez grado 3 & 8 & 6.7 & 8 & 10.7 & 0 & 0 \\
\hline Delgadez grado $2^{\mathrm{a} *}$ & 4 & 3.3 & 4 & 5.3 & 0 & 0 \\
\hline Delgadez grado 1 & 8 & 6.7 & 5 & 6.7 & 3 & 6.7 \\
\hline Normal & 82 & 68.3 & 51 & 68 & 31 & 68.9 \\
\hline Sobrepeso & 14 & 11.7 & 6 & 8 & 8 & 17.8 \\
\hline Obesidad & 4 & 3.3 & 1 & 1.3 & 3 & 6.7 \\
\hline
\end{tabular}

En cuanto al análisis de las diferencias de medias de las variables antropométricas por localidad se observó diferencia estadísticamente significativa $(\mathrm{p}=0.05)$ en cuanto al IMC, siendo la localidad de Xadani la de menor IMC (Tabla 2).

Tabla 2. Comparación de medias de variables antropométricas

\begin{tabular}{|c|c|c|c|c|c|c|}
\hline \multirow{3}{*}{ Variable } & \multirow{2}{*}{\multicolumn{2}{|c|}{$\begin{array}{c}\text { Total } \\
\mathrm{N}=120\end{array}$}} & \multirow{2}{*}{\multicolumn{2}{|c|}{$\frac{\text { Xadani }}{\mathrm{N}=75}$}} & \multirow{2}{*}{\multicolumn{2}{|c|}{$\begin{array}{c}\text { Progreso } \\
N=45\end{array}$}} \\
\hline & & & & & & \\
\hline & Media & $\pm \mathrm{DE}$ & Media & $\pm \mathrm{DE}$ & Media & $\pm \mathrm{DE}$ \\
\hline Talla $(\mathrm{cm})^{\mathrm{a}}$ & 130.53 & 12.87 & 131.03 & 12.54 & 129.69 & 13.49 \\
\hline Peso $(\mathrm{kg})^{\mathrm{b}}$ & 28.79 & 9.44 & 27.75 & 8.45 & 30.51 & 10.75 \\
\hline $\operatorname{IMC}\left(\mathrm{kg} / \mathrm{m}^{2}\right)^{\mathrm{b}_{*}}$ & 16.58 & 3.1 & 15.96 & 2.97 & 17.59 & 3.03 \\
\hline \multicolumn{7}{|c|}{ Fuente: Elaboración propia con datos obtenidos en el programa SPSS V. 2} \\
\hline \multicolumn{7}{|c|}{${ }^{\mathrm{a}}$ Prueba t student. } \\
\hline \multicolumn{7}{|c|}{${ }^{\mathrm{b}}$ Prueba Mann-Whitney. } \\
\hline \multicolumn{7}{|c|}{ DE: desviación estándar. } \\
\hline \multicolumn{7}{|c|}{${ }^{*}$ Significancia $(p<0.05)$} \\
\hline
\end{tabular}

En cuanto al indicador Talla/E, solo el 3.3\% (4) presentó un retraso en el crecimiento, sin mostrar diferencias estadísticamente significativas entre comunidades ( $>0.05)$. Cabe resaltar que los casos anteriormente mencionados, tuvieron un diagnóstico de acuerdo con el IMC/E normal. Del resto de niños el $95.8 \%$ presentaron talla normal y el $0.8 \%$ (1) talla alta para la edad. Tampoco se mostraron diferencias estadísticamente significativas de Talla/edad entre niños y niñas ( $p>0.05)$. (Datos no mostrados).

En cuanto al indicador IMC/E en la categoría de desnutrición se observó una diferencia estadísticamente significativa $(\mathrm{p}<0.05)$; así como cuatro veces la probabilidad $(\mathrm{OR}$ crudo $=4.10 \mathrm{IC}$ 95\% 1.13-14.91) de padecer algún grado de delgadez al proceder de la comunidad de Xadani en comparación a la localidad de progreso. Con respecto al sobrepeso y la obesidad no se observaron diferencias significativas entre ambas localidades.

Tabla 3. Asociación del estado nutricional por localidad

\begin{tabular}{|c|c|c|c|c|c|}
\hline \multirow{2}{*}{ Variable } & \multicolumn{2}{|c|}{ Xadani } & \multicolumn{2}{|c|}{ Progreso } & \multirow{2}{*}{$\mathrm{OR}^{\mathrm{b}}$} \\
\hline & 75 & $\%$ & 45 & $\%$ & \\
\hline \multicolumn{6}{|l|}{ Talla/Edad } \\
\hline Talla baja ${ }^{a}$ & 3.0 & 2.5 & 1.0 & 2.1 & 1.83 (IC:0.18-18.18) \\
\hline Normal $^{\mathrm{a}}$ & 71 & 94.7 & 44 & 97.9 & 0.41 (IC:0.04-3.73) \\
\hline Muy alto ${ }^{c}$ & 1.0 & 1.3 & 0 & 0 & 1.61 (IC:1.40-1.85) \\
\hline \multicolumn{6}{|l|}{ IMC/Edad } \\
\hline Desnutrición $^{a *}$ & 17 & 22.7 & 3.0 & 6.7 & 4.10 (IC: $1.13-14.91)$ \\
\hline Normal $^{\mathrm{a}}$ & 51 & 62.2 & 31.0 & 37.8 & 1.04 (IC: 0.47-2.31) \\
\hline Sobrepeso ${ }^{a}$ & 6.0 & 8.0 & 8.0 & 17.8 & 0.40 (IC: 0.13-1.25) \\
\hline Obesidad $^{\mathrm{a}}$ & 1.0 & 1.3 & 3.0 & 6.7 & 0.19 (IC: 0.02-1.88) \\
\hline \multicolumn{6}{|c|}{ Fuente: Elaboración propia con datos obtenidos en el programa SPSS V. 23} \\
\hline \multicolumn{6}{|l|}{${ }^{a}$ Chi-cuadrada. } \\
\hline \multicolumn{6}{|l|}{${ }^{\mathrm{b}}$ OR: Crudo } \\
\hline${ }^{\mathrm{c}}$ Prueba de Fis & & & & & \\
\hline
\end{tabular}

En la tabla 4 se puede observar la comparación del estado nutricional de dos grupos estratificados de cada localidad, el grupo 1 conformado por alumnos de $1^{\circ}$ a $3^{\circ}$ y el grupo 2 por escolares de $4^{\circ}$ a $6^{\circ}$, en cuanto a la Talla/E no se mostraron diferencias importantes entre cada grupo; por otra parte de acuerdo al IMC/E se evidenció una diferencia estadísticamente significativa $(\mathrm{p}<0.05)$ entre grupos de la comunidad de Xadani, de este modo se puede observar una mayor proporción de delgadez en el grupo 2, respecto al grupo contrario ; así como una mayor frecuencia de normalidad en el grupo 1, con respecto al grupo 2 . 
Tabla 4. Estado nutricional por localidad y grupo de estratificación

\begin{tabular}{|c|c|c|c|c|c|c|c|c|}
\hline \multirow{3}{*}{$\begin{array}{c}\text { Estado } \\
\text { Nutricional }\end{array}$} & \multicolumn{4}{|c|}{ Xadani } & \multicolumn{4}{|c|}{ Progreso } \\
\hline & \multicolumn{2}{|c|}{ Grupo 1} & \multicolumn{2}{|c|}{ Grupo 2} & \multicolumn{2}{|c|}{ Grupo 1} & \multicolumn{2}{|c|}{ Grupo 2} \\
\hline & $\mathrm{N}=32$ & $\%$ & $\mathrm{~N}=43$ & $\%$ & $\mathrm{~N}=26$ & $\%$ & $\mathrm{~N}=19$ & $\%$ \\
\hline \multicolumn{9}{|l|}{$\overline{\text { Talla/Edad }^{a}}$} \\
\hline Baja & 3 & 9.4 & 0 & 0 & 1 & 3.8 & 0 & 0 \\
\hline Normal & 29 & 90.6 & 42 & 97.7 & 25 & 96.2 & 19 & 100 \\
\hline Muy alto & 0 & 0 & 1 & 2.3 & 0 & 0 & 0 & 0 \\
\hline \multicolumn{9}{|l|}{${\mathrm{IMC} / \mathrm{Edad}^{\mathrm{a} *}}$} \\
\hline Desnutrición & 1 & 3.1 & 16 & 37.2 & 2 & 7.7 & 1 & 5.3 \\
\hline Normal & 31 & 96.9 & 20 & 46.5 & 18 & 69.2 & 13 & 68.4 \\
\hline Sobrepeso & 0 & 0 & 6 & 14 & 4 & 15.4 & 4 & 21.1 \\
\hline Obesidad & 0 & 0 & 1 & 2.3 & 2 & 7.7 & 1 & 5.3 \\
\hline \multicolumn{9}{|c|}{ Fuente: Elaboración propia con datos obtenidos en el programa SPSS V. 23} \\
\hline \multicolumn{9}{|c|}{ Grupo 1: Alumnos de $1^{\circ}$ a $3^{\circ}$} \\
\hline \multicolumn{9}{|c|}{ Grupo 2: Alumnos de $4^{\circ}$ a $6^{\circ}$} \\
\hline \multicolumn{9}{|l|}{ a Chi-cuadrada. } \\
\hline *Significancia & 0 & & & & & & & \\
\hline
\end{tabular}

\section{Discusión}

Uno de los grupos poblacionales más susceptibles a variaciones en su estado nutricional es el grupo de escolares. Este grupo etario se ve influenciado por diversos factores económicos, demográficos, sociales y biológicos, los cuales pueden llevarlos a un estado de desnutrición u obesidad. (Golden, 1994) Dentro de los aspectos sociales, una variable importante a considerar es la condición indígena, ya que de acuerdo con lo descrito por lo Organización de las Naciones Unidas para la Agricultura y Alimentación (FAO,2013) las poblaciones rurales e indígenas de América Latina siguen siendo las más vulnerables por su alto nivel de pobreza, discriminación, menor acceso a la educación y la sanidad, incluido el aspecto alimentario y nutricional. En este mismo sentido, la UNICEF (2014) reporta que 9 de cada 10 niños que hablan alguna lengua indígena son pobres, por lo cual, se podría decir que un niño indígena en edad escolar es más susceptible a problemas de salud, alimentación y nutrición.

En el presente estudio el estado de nutrición predominante en ambas localidades indígenas fue de normalidad con el $68.3 \%$ (82) en toda la población analizada. La prevalencia conjunta de desnutrición en ambas localidades fue de $16.7 \%$, mostrándose superior que la de SP + OB con un $15 \%$.

Estos datos contrastan con los encontrados en otro estudio realizado en niños escolares asignados al Centro de Salud con Servicios Ampliados (CESSA) del municipio de Tlalixtac de Cabrera, Oaxaca el cual tiene características sociales similares a la población estudiada, encontrando una prevalencia de $7 \%$ de desnutrición y $44 \%$ de SP+OB, (Monroy, 2016) muy por arriba de lo referido en nuestros resultados. En cuanto a la prevalencia de desnutrición, las cifras son similares a las de un estudio de una cohorte de 960 niños en Chihuahua de escuelas rurales en donde se encontró una prevalencia de desnutrición de $14.6 \%$ y de SP+OB de 15.4\% (Martínez et al.,2017). También se aproximan a las reportadas en el Registro Nacional de Peso y Talla (RNPT) de estados con características sociodemográficas similares como Guerrero y Chiapas con un $19.4 \%$ y $12.4 \%$ respectivamente (Âvila, 2016).

Por otro lado, las cifras de SP + OB y estado normal de nutrición son semejantes a las encontradas en un estudio de colonias rurales de Oaxaca, con una prevalencia de OB + SP de $12 \%$ y de $66 \%$ de estado de nutrición normal. (Malina, et al., 2011). También se asemejan a las de otro estudio realizado en comunidades rurales del municipio de Ixhuatlán de Madero, Veracruz, con cifras de SP + OB de $17.8 \%$. (García et al., 2017) y a la de otro estudio de tres comunidades rurales de Yucatán, en donde se obtuvo una prevalencia de $\mathrm{SP}+\mathrm{OB}$ de $16.4 \%$ (Azcorra et al., 2016). Sin embargo, la prevalencia de SP+OB encontrada en nuestro estudio (15\%) está muy por debajo de lo reportado en la ENSANUT 2012 en Oaxaca con un $27.5 \%$ o la reportada por el RNPT en 2016 con un $31.7 \%$ para el mismo estado y grupo poblacional (Ávila, 2016) y por debajo a nivel nacional de lo que estimó la ESANUT MC 2016 con una prevalencia conjunta SP + OB de 33.2\%. (Shammah, 2018).

Diversos estudios realizados en población escolar reportan que aquellos niños con un medio y situación de empobrecimiento tienen mayor acceso a alimentos densamente energéticos con un pobre contenido nutricional y que el estado nutricional de escolares está condicionado a la calidad de vida (Lee,2012). Esto es consistente con un análisis sobre costos por caloría en México en el cual se encontró que los patrones de consumo difieren de acuerdo al nivel de ingreso, en donde los hogares con un menor ingreso toman decisiones de gasto que les permiten consumir una mayor cantidad de calorías a un menor precio, pero de baja calidad nutricional; mientras que en los hogares de ingresos altos se consumieron alimentos de mayor costo por caloría (Hernández, 
2012). Sin embargo, estos datos en determinado momento pueden ser relativos y podrían explicar las variaciones entre estudios y resultados contradictorios, ya que se hace evidente que no todas las zonas rurales y pobres de México obedecen este comportamiento generalizado asociado a la transición nutricional y epidemiológica. La variabilidad de las cifras de delgadez, SP y OB también pueden sugerir la influencia del área geográfica, tipo de municipio y la avanzada transición nutricional en aquellos lugares en donde las prevalencias de $\mathrm{SP}+\mathrm{OB}$ son alarmantes, sin embargo, en los resultados de este estudio tanto la delgadez como el SP y la OB estuvieron presentes en similitud de proporciones, dejándose notar la doble carga de los problemas carenciales y de exceso, lo cual sugiere que las comunidades analizadas quizá no tienen acceso a los alimentos altamente energéticos e industrializados a razón de la pobreza, a pesar de la disponibilidad global de los mismos, o que están en plena transición nutricional y epidemiológica aumentando su prevalencia de SP+OB. Desafortunadamente no se cuentan con datos previos para contrastar nuestros resultados. A pesar de esto, la coexistencia de estas condiciones nutricionales indica la necesidad de replantear políticas y programas en torno a la noción de alimentación saludable y estilos de vida saludables en México. (Rivera et al., 2014)

Si bien, otro de los puntos débiles en la presente investigación fue que no se evaluó el consumo de alimentos, un estudio realizado en la ciudad de Oaxaca en el año 2000 ,evalúo la distribución de energía en condiciones de bajos ingresos en niños de 4 a 6 años, la cual reflejó que el 10\% de la energía total provenía de proteínas, $78 \%$ de carbohidratos y $12 \%$ de grasas; no obstante la población de las dos comunidades estudiadas en esta investigación cuentan con características sociodemográficas en mayor desventaja que las estudiadas por los autores; por lo tanto se podrían esperar valores más alterados, principalmente bajo consumo de proteína, siendo un macronutriente esencial para el crecimiento y desarrollo en la edad escolar. (Wyatt y Triana, 2000).

Esto resalta aún más cuando se hizo el análisis por localidad, ya que Xadani sí mostró diferencia estadísticamente significativa con cifras más elevadas de delgadez que Progreso $(\mathrm{p}=0.02)$, así como cuatro veces mayor probabilidad $(\mathrm{OR}$ crudo $=$
4.10 IC: 95\% 1.13-14.91) de presentar algún grado de delgadez y no solo eso, también mostró la mayor cantidad de niños con talla baja, siendo esta una localidad más pobre (SEDESOL, 2013). Asimismo, mostró una media de IMC menor a la de Progreso. Independientemente que la prevalencia de talla baja para la edad no es elevada en la población estudiada, no hay que descartar su importancia, ya que, a pesar de que se ha observado que la población escolar durante su desarrollo hacía la edad adulta puede experimentar algún grado de recuperación espontanea en su talla, principalmente a razón de la modificación del medio ambiente en el que se desarrollan y de la alimentación, se esperarían variaciones en sus potenciales de altura máximos (Golden, 1994), motivo de preocupación debido al efecto adverso irreversible en el desarrollo físico e intelectual de los niños (Black et al., 2008).

Cuando se realizó el análisis por grupos estratificados de cada localidad, se pudo observar diferencia estadísticamente significativa $(\mathrm{p}=0.001)$ entre los grupos de Xadani, siendo los de $4^{\circ}$ a $6^{\circ}$ los que presentaron mayor probabilidad de presentar algún grado de delgadez. Este resultado tiene semejanza con un estudio realizado en escolares de los municipios Libertador, Campo Elías, Santos Marquina y Sucre del estado de Mérida (Venezuela) en escolares de $1^{\circ}$ a $6^{\circ}$ grado, en donde se presentó mayor prevalencia de déficit según el peso para la edad en $5^{\circ}$ y $6^{\circ}$ grado (Angulo, 2013). Esto posiblemente puede ser ocasionado por el aumento de los requerimientos nutricionales relacionados con el crecimiento natural ya que en nuestro estudio la mayoría de los niños presentaron una talla normal (95.8\%) para la edad en las dos localidades, descartando la desnutrición crónica y favoreciendo la hipótesis de que posiblemente en etapas más tempranas los requerimientos nutricionales y energéticos pudieron ser cubiertos adecuadamente y conforme la edad aumenta y la composición corporal cambia, se vuelve más difícil cubrir el consumo energético-nutricional en un grado óptimo propiciando el bajo peso, ya que el aumento de los requerimientos energéticos puede ser considerable con la edad y con la actividad física hasta en $600 \mathrm{kcal}$ o más entre niños de 4 y 13 años (Hidalgo y Güemes, 2011). Esto también se relaciona con el hecho de que a nivel mundial se estima que un $10 \%$ de los niños de 5 a 19 años tiene un IMC para la edad dos desviaciones estándar inferior a la media de la 
población de referencia de la OMS y, existen notables diferencias en la prevalencia de la delgadez entre los niños de 5 a 19 años en diferentes regiones del mundo que en general se mantiene igual desde el año 2005 (FAO, FIDA, UNICEF y OMS, 2018). Esto puede estar condicionado por la capacidad adquisitiva de las familias y la estructura de las mismas. Lamentablemente y a pesar de que ambas localidades se consideran con alto grado de marginación y pobreza en nuestro estudio no se obtuvieron datos socioeconómicos, por lo cual no es posible establecer con claridad la relación entre estado nutricional y condiciones socioeconómicas de los niños y de sus familias.

Aunque este estudio presenta algunas debilidades metodológicas como se han ido mencionando con anterioridad, y que las asociaciones encontradas son hasta cierto punto débiles, podría ser un referente para nuevas investigaciones y nos da indicios para valorar nuevas variables y profundizar en el tema y así poder explicar la posible consecuencia de la prevalencia de malnutrición presentada en esta población ya que es la primera investigación que se lleva a cabo en este tipo de localidades, que muchas veces son olvidadas y que viven en una situación de desventaja en todos los aspectos sociales.

\section{Conclusiones}

Es un hecho que la malnutrición tiene diferentes manifestaciones y que en México aún coexisten la desnutrición, el sobrepeso y la obesidad. Desde esta perspectiva, está ampliamente documentado que la transición nutricional y epidemiológica ha permeado en las zonas rurales y en población indígena aumentando las cifras de sobrepeso y obesidad como se menciona en la ENSANUT MC 2016, sin embargo, se ha demostrado que aún existen zonas cuyo nivel de pobreza es condicionante y juega un papel fundamental en la presencia de desnutrición, ya que la disponibilidad de alimentos altamente energéticos e industrializados principalmente en las zonas rurales no garantiza su accesibilidad, es decir, su adquisición o consumo ya que este aspecto está relacionado al ámbito económico. La doble carga de morbilidad de problemas nutricionales es característica de países en desarrollo como México, y este doble reto condiciona una duplicidad de esfuerzos y recursos, sin embargo, son condiciones prevenibles. En este estudio se pudo dar por sentado que la población de las localidades de Progreso y Xadani son un ejemplo de la doble carga de problemas nutricionales polarizados en deficiencias y excesos; no obstante la distribución de la misma no es homogénea, debido a que, los escolares de Xadani tienen mayor probabilidad de presentar delgadez en comparación a la comunidad aledaña lo que nos lleva a reflexionar nuevas variables de estudio que expliquen la heterogeneidad dentro de un mismo contexto; de igual manera conocer los factores inmersos en la delgadez presente en escolares de mayor edad en donde el aumento de sus requerimientos de nutrimientos es mayor $\mathrm{y}$ evidentemente no se llegan a cumplir, en ambos casos se considera que la seguridad alimentaria ejercer un papel importante ante esta situación. Este tipo de investigaciones evidencian y refuerzan la necesidad de intervenir nutricionalmente por parte de las autoridades sanitarias, a través de programas escolares como una plataforma para el suministro de alimentos y suplementos que puedan contribuir a la mejora de su estado nutricional.

\section{Agradecimientos}

Se agradece a los niños participantes, así como a las autoridades de las instituciones educativas que permitieron el presente estudio.

\section{Bibliografía}

Angulo L. (2013). Diagnóstico nutricional en escolares de los municipios Libertador, Campo Elías, Santos Marquina y Sucre del estado Mérida. Educere, 58, 515-526. Recuperado de: https://www.redalyc.org/articulo.oa?id=3563040 4015

Arcaya M., Arcaya A. y Subramanian S. (2015). Desigualdades en salud: definiciones, conceptos y teorías. Rev Panam Salud Publica, 38(4), 261271. DOI: $10.3402 /$ gha. v8.27106.

Avila A., Galindo C., Juárez L., Del Monte M. y Ávila M. (2015). Manual de antropometría, Registro Nacional de Peso y Talla en Escolares. Recuperado de: http://rnpt.sivne.org.mx/web/documentos/manua les_y_formatos/MANUALANTROPOMETRIA .pdf)

Badillo P., Rodríguez M. y Nieves X. (2017). Impact of Metabolic Hormones Secreted in Human 
Breast Milk on Nutritional Programming in Childhood Obesity. J Mammary Gland Biol Neoplasia, 22(3), 171.191. DOI: 10.1007/s10911-017-9382-y

Black R., Allen L., Bhutta Z., Caulfield L., De Onis M., Ezzati M, et al. (2008). Maternal and child undernutrition: global and regional exposures and health consequences. Lancet, 371:243-60. DOI: 10.1016/S0140-6736(07)61690-0

Bouma S. (2017). Diagnosing Pediatric Malnutrition: Paradigm Shifts of EtiologyRelated Definitions and Appraisal of the Indicators. Nutr Clin Pract, 32(1),52-67. DOI: $10.1177 / 0884533616671861$

Dinkel D., Hanson C., Koehler K., Anderson A., Kyvelidou A., Bice M., Wallen J., Bagenda D., Jana L. y Pressler J. (2018). An overview of assessment methodology for obesity-related variables in infants at risk. Nutr Health, 24(1), 113.DOI: $10.1177 / 0260106017732268$

Endris, N., Asefa, H., y Dube, L. (2017). Prevalence of Malnutrition and Associated Factors among Children in Rural Ethiopia. BioMed Research International, 2017,1-6. DOI: $10.1155 / 2017 / 6587853$

FAO, FIDA, UNICEF, PMA y OMS. (2018). El estado de la seguridad alimentaria y la nutrición en el mundo. Fomentando la resiliencia climática en aras de la seguridad alimentaria y la nutrición. Recuperado de: https://www.who.int/nutrition/publications/foods ecurity/state-food-security-nutrition-2018-es.pdf

García C., González E., Meléndez J., García P. y García C. (2017). Estudio de la situación nutricional y hábitos alimentarios de escolares de diferentes comunidades indígenas del municipio de Ixhuatlán de Madero, Estado de Veracruz (México), Archivos Latinoamericanos de Nutrición, 67(4),238-250. Recuperado de: https://www.alanrevista.org/ediciones/2017/4/art $-1 /$

Golden M. (1994). Is complete catch-up possible for stunted malnourished children? Eur J Clin Nutr,
48(1), 58-70. Recuperado de: https://www.ncbi.nlm.nih.gov/pubmed/8005092

Hernández G., Minor, E., Aranda, R. (2012). "Determinantes Económicos: Evolución del Costo de las Calorías en México". Obesidad en México: Recomendaciones Para una Política de Estado. UNAM. Pp. 145-164. Recuperado de: https://www.anmm.org.mx/publicaciones/Obesi dad/obesidad.pdf

Hidalgo M. y Guemes M. (2011). Nutrición del preescolar, escolar y adolescente. Pediatr Integral, 15(4), 351-368. Recuperado de: http://nutria01.emiweb.es/medias/files/pediatriaintegral-xv-4.pdf\#page $=52$

IMB Corp. (2016). IMB SPSS Statistics for windows. Versión 24.0. Armonk NY: IMB Corp. Recuperado de: https://www.ibm.com/support/pages/how-citeibm-spss-statistics-or-earlier-versions-spss

Instituto Nacional de Pueblos Indígenas. (2015). Indicadores de Población Indígena: catálogo de localidades Indígenas. Recuperado de: https://www.gob.mx/inpi/documentos/indicador es-de-la-poblacion-indigena

Kruger H., Steyn N., Maunder E., Neil J., Moeng L., et al. (2012). Overweight among children decreased, but obesity prevalence remained high among women in South Africa, 1999-2005. Public Health Nutr, 15(4),594-599. DOI: 10.1017/S136898001100262X

Lee H. (2012). The role of local food availability in explaining obesity risk among young school-aged children. Social Science \& Medicine, 74(8), 1193-1203.

DOI: 10.1016/j.socscimed.2011.12.036

Macari M., Berumen J. y Calvillo A. (2018). Alianza por la Salud Alimentaria: Publicidad dirigida a niños: una infancia enganchada a la obesidad. Recuperado de: https://alianzasalud.org.mx/wpcontent/uploads/2018/08/d-publicidad-dirigidaa-ninios-una-infancia-enganchada-a-laobesidad-b.pdf 
Mameli C., Mazzantini S y Vincenzo G. (2016). Nutrition in the First 1000 Days: The Origin of Childhood Obesity. En t. J. Environ. Res. Salud Pública, 13(9), 838; DOI:10.3390/ijerph13090838

Martínez L., Viloria, M., Nájera, A., Urquidez R. y Ramos A. (2017). Estado nutricional en escolares de una zona mediorural del norte de México que reciben apoyo alimentario. revista de ciencia y tecnología de la UACJ, 15(1), 29-38. Recuperado de:

http://erevistas.uacj.mx/ojs/index.php/cienciafro ntera/article/view/2489/2307

Monroy G., González MA., Pérez I., Matías D y García IA. Características antropométricas y hábitos dietéticos en niños de etapa escolar del centro de salud con servicios ampliados de Tlalixtac de Cabrera, Oaxaca, México. Nutr. clín. diet. hosp. 2016; 36(3):189-193.

Pollitt E., Gorman K., Engle P., Rivera J y Martorell R. (1995). Nutrition in early life and the fulfillment of intellectual potential. $J \mathrm{Nutr}$, 125(4), 1111-1118. DOI: 10.1093/jn/125.suppl_4.1111S

Rivera J., Pedraza L., Martorell r. y Gil A. (2014). Introduction to the double burden of undernutrition and excess weight in Latin America. Am J Clin Nutr, 100(6), 1613-1616. DOI: doi.org/10.3945/ajcn.114.084806

SEDESOL (2013). Catálogo de Localidades. Sistema de apoyo para la Planeación del PDZP. Recuperado de: http://www.microrregiones.gob.mx/catloc/conte nido.aspx ?refnac $=200360018$

SEDESOL. (2013). Catálogo de Localidades. Sistema de apoyo para la Planeación del PDZP. Recuperado de: http://www.microrregiones.gob.mx/catloc/conte nido.aspx?refnac $=200360020$

SEDESOL. (2013). Catálogo de localidades: Sistema de Apoyo para la Planeación del Programa para el Desarrollo de Zonas Prioritarias

(PDZP)
http://www.microrregiones.gob.mx/catloc/Defau lt.aspx $?$ tipo $=$ clave $\&$ campo $=$ mun $\&$ valor $=20$

Shama T., Amaya M. y Cuevas L. (2015). Desnutrición y obesidad: doble carga en México. Revista Digital Universitaria, 16(5). Recuperado de:

http://www.revista.unam.mx/vol.16/num5/art34/ art34.pdf

Shamah T., Cuevas L., Gaona EB., Gómez LM., Morales M., Hernández M, et al. (2018). Sobrepeso y obesidad en niños y adolescentes en México, actualización de la Encuesta Nacional de Salud y Nutrición de Medio Camino 2016. Salud Pública Mex, 60(3):244-256. DOI: $10.21149 / 8815$

StataCorp (2015). Stata Statistical Software: Release 14. College Station, TX: stata corp. Recuperado de:

https://www.stata.com/support/faqs/resources/cit ing-software-documentation-faqs/

UNICEF. (2012). Evaluación del crecimiento de niños y niñas. Material de apoyo para equipos de atención primaria de la salud. Recuperado de: http://files.unicef.org/argentina/spanish/Nutricio n_24julio.pdf

UNICEF. (2014). Pobreza y derechos sociales de niñas, niños y adolescentes POBR en México, 2014. Recuperado de: https://www.coneval.org.mx/Medicion/Docume nts/Estudio-Pobreza-Coneval-Unicef.pdf

UNICEF. (2016). Salud y Nutrición. Recuperado de: https://www.unicef.org/mexico/salud-ynutrición

Wyatt C. y Triana M. (2000). Nutrient Intake and Growth of Preschool Children from Different Socioeconomic Regions in the City of Oaxaca, México. Ann Nutr Metab, 44, 14-20. DOI: $10.1159 / 000012816$ 